\title{
RESPOSTA DO ARROZ IRRIGADO À ADUBAÇÃO RESIDUAL E AOS NÍVEIS DE ADUBAÇÃO EM SOLO DE VÁRZEA
}

\author{
Nand Kumar Fageria ${ }^{1}$, Alberto Baêta dos $\operatorname{Santos}^{2}$ \& \\ Francisco José P. Zimmermann ${ }^{1}$
}

\begin{abstract}
RESUMO
Informações sobre o efeito residual da adubação na produção de arroz irrigado em solos de várzeas no Brasil, são escassas. Conduziu-se um experimento de campo, durante três anos consecutivos, para se avaliar o efeito residual da adubação e de níveis de adubação aplicados no sulco de plantio sobre a produção de arroz irrigado em um solo Glei Pouco Húmico (Inseptissolo) de várzea, período em que a produção de matéria seca da parte aérea e de grãos foi significativamente afetada pela adubação no sulco e não pela adubação residual. As produções máximas de matéria seca e de grãos foram obtidas com o tratamento $T_{7}$ (efeito residual da adubação média + adubo verde $+75 \mathrm{~kg} \mathrm{~N} \mathrm{ha}^{-1}, 90 \mathrm{~kg}$ $\mathrm{P}_{2} \mathrm{O}_{5}$ ha $^{-1}$ e $60 \mathrm{~kg} \mathrm{~K}_{2} \mathrm{O} \mathrm{ha}^{-1}$ ). O tratamento $\mathrm{T}_{4}$ (efeito residual da adubação média $+50 \mathrm{~kg} \mathrm{~N} \mathrm{ha}^{-1}, 60 \mathrm{~kg} \mathrm{P}_{2} 0_{5}$ $\left.\mathrm{ha}^{-1} \mathrm{e} 40 \mathrm{~kg} \mathrm{~K}_{2} 0 \mathrm{ha}^{-1}\right)$ não foi diferente significativamente em comparação com o tratamento $\mathrm{T}_{7}$; enquanto entre os componentes da produção o número de panículas por $\mathrm{m}^{2}$ e o peso de 1.000 grãos foram significativamente afetados pelos tratamentos, por outro lado, a acumulação da maioria dos nutrientes na parte aérea e nos grãos foi significativamente afetada pelos tratamentos.
\end{abstract}

Palavras-chave: acumulação de nutrientes, Oryza sativa, propriedades químicas do solo

\section{RESPONSE OF IRRIGATED RICE TO RESIDUAL FERTILIZATION AND TO APPLIED LEVELS OF FERTILIZERS IN LOWLAND SOIL}

\begin{abstract}
Information on the residual effect of fertilization is scarce for irrigated rice produced in lowland soils in Brazil. A field experiment was conducted during three consecutive years to evaluate the effects of residual and band applied fertilizers on irrigated rice yield in a Low Humic Gley Soil (Inceptisol). Mean dry matter and grain yield were significantly affected with applied fertilizer treatments, which did not happen for residual fertilizer treatments. Maximum dry matter and grain yield were produced under treatment which consisted of residual effect of medium soil fertility level plus green manure $+75 \mathrm{~kg} \mathrm{~N}$ $\mathrm{ha}^{-1}, 90 \mathrm{~kg} \mathrm{P}_{2} \mathrm{O}_{5} \mathrm{ha}^{-1}$, and $60 \mathrm{~kg} \mathrm{~K}_{2} \mathrm{O} \mathrm{ha}^{-1}\left(\mathrm{~T}_{7}\right)$. The treatment of residual effect of medium soil fertility level plus $50 \mathrm{~kg} \mathrm{~N} \mathrm{ha}^{-1}, 60 \mathrm{~kg} \mathrm{P}_{2} \mathrm{O}_{5} \mathrm{ha}^{-1}$ and $40 \mathrm{~kg} \mathrm{~K}_{2} \mathrm{O} \mathrm{ha}^{-1}\left(\mathrm{~T}_{4}\right)$ did not show significant differences in comparison to $\mathrm{T}_{7}$ treatment. Number of panicles $\mathrm{m}^{-2}$ and 1000 grains weight were the yield components which were significantly affected with fertilizer treatments. Accumulation of almost all the nutrients in the dry matter and grain were significantly affected with fertilizer treatments.
\end{abstract}

Key words: nutrient accumulation, Oryza sativa, soil chemical properties

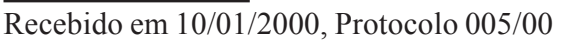

${ }^{1}$ Eng. Agr., Ph.D., Embrapa Arroz e Feijão, Bolsista do CNPq, CP 179, CEP 75375 - 000 Santo Antônio de Goiás, GO.

Fone: (0xx62) 533 2178, Fax: (0xx62) 533 2100.E-mail: fageria@cnpaf.embrapa.br

${ }^{2}$ Eng. Agr., Dr., Embrapa Arroz e Feijão 


\section{INTRODUÇ̃̃O}

A agricultura moderna exige o uso de fertilizantes e corretivos em quantidades suficientes para aumentar a produtividade e, ao mesmo tempo, manter a fertilidade do solo (van Raij, 1991; Fageria, 1992). Existem, no Brasil, cerca de 30 milhões de hectares de várzea e sua maioria ainda não foi utilizada (Fageria, 1999b). Em solo de várzea, foi comparada a importância relativa dos nutrientes N, P e K, na produção de arroz irrigado (Fageria, 1999a). Com base nesses dados, conclui-se que o $\mathrm{N}$ é mais limitante para a produção de arroz, seguido do P e do K. Para se obter alta produção nesses solos é necessário corrigir-se a deficiência nutricional e, no manejo adequado de sua fertilidade, o efeito residual da adubação é importante, principalmente de nutrientes imóveis como P e K. Fageria et al. (1999) definiram o efeito residual do fósforo e do potássio como a quantidade desses nutrientes disponível para a cultura sucessiva ou a resposta desta cultura a nível de $\mathrm{P}$ e $\mathrm{K}$ aplicados. O efeito residual depende do tipo do solo, da quantidade de nutrientes aplicados e da produtividade.

O uso racional da adubação não somente aumenta a produtividade das culturas como, também, diminui o custo de produção e os riscos de poluição ambiental. Neste sentido, a avaliação do efeito residual da adubação é fundamental para o manejo da fertilidade do solo na produção de culturas anuais, como o arroz irrigado. São escassas as informações sobre este assunto; por este motivo, foi conduzido um experimento de campo, com o objetivo de se avaliar o efeito residual e de níveis de adubação aplicados no sulco de plantio sobre a produção de arroz irrigado, em solo de várzea. O conhecimento do efeito residual da adubação é de grande importância, considerando-se a necessidade de racionalizar o uso de fertilizantes e de aumentar a eficiência nutricional da cultura de arroz irrigado.

\section{MATERIAL E MÉTODOS}

O experimento foi conduzido na Fazenda Palmital, da Embrapa Arroz e Feijão, localizada no município de Goianira, GO, durante três anos consecutivos. O solo da área experimental é classificado como Glei Pouco Húmico (Inceptisssolo) de várzea e sua análise química apresentou as seguintes características: pH em água 4,8 (1:2,5); M.O. $14 \mathrm{~g} \mathrm{~kg}^{-1}$; P 16,5 mg kg-1; K 80 mg

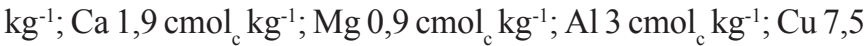

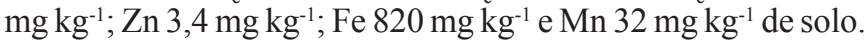
Os $\mathrm{P}, \mathrm{K}, \mathrm{Zn}, \mathrm{Cu}, \mathrm{Fe}$ e Mn, foram extraídos pelo extrator Mehlich $1\left(0,05 \mathrm{M} \mathrm{HCl}+0,0125 \mathrm{M} \mathrm{H}_{2} \mathrm{SO}_{4}\right)$, os $\mathrm{Ca}, \mathrm{Mg}$ e Al o foram com solução de $\mathrm{KCl} 1 \mathrm{M}$. Na solução extraída, o P foi determinado por colorimetria, o K por fotometria de chama e os $\mathrm{Cu}, \mathrm{Zn}$, Fe e Mn por absorção atômica. O Ca e o Mg na solução extraída foram determinados por titulação com EDTA e o Al por titulação com $\mathrm{NaOH}$. A matéria orgânica foi determinada pelo método de Walkley-Black e a metodologia de análise está descrita no Manual de Métodos de Análise do Solo da Embrapa (1997).

Anteriormente à instalação do experimento foi realizado, na mesma área, estudo com arroz e feijão em rotação, por três anos consecutivos $\mathrm{O}$ primeiro cultivo foi de arroz, com três níveis de adubação: 1) testemunha (sem adubação- fertilidade natural do solo); 2) $100 \mathrm{~kg} \mathrm{~N} \mathrm{ha}^{-1}, 100 \mathrm{~kg} \mathrm{P}_{2} \mathrm{O}_{5} \mathrm{ha}^{-1}, 60 \mathrm{~kg} \mathrm{~K}_{2} \mathrm{O} \mathrm{ha}^{-1}, 40 \mathrm{~kg}$ FTE-BR-12 ha-1 (nível médio de adubação); 3) $200 \mathrm{~kg} \mathrm{~N} \mathrm{ha}^{-1}$,
$200 \mathrm{~kg} \mathrm{P}_{2} \mathrm{O}_{5}$ ha $^{-1}, 120 \mathrm{~kg} \mathrm{~K}_{2} \mathrm{O} \mathrm{ha}^{-1}, 80 \mathrm{~kg} \mathrm{FTE-BR-12} \mathrm{ha-1} \mathrm{(nível}$ alto de adubação); 4) $28 \mathrm{t} \mathrm{ha}^{-1}$ de massa fresca de feijão guandu (Cajanus cajan L.). Este adubo verde foi incorporado ao solo por ocasião da colheita da primeira safra de arroz.

Na cultura do feijoeiro produzido no inverno com irrigação suplementar nas mesmas parcelas de arroz, a adubação consistiu em: 1) testemunha (sem adubação-fertilidade natural do solo); 2) $35 \mathrm{~kg} \mathrm{~N} \mathrm{ha}^{-1}, 120 \mathrm{~kg} \mathrm{P}_{2} \mathrm{O}_{5} \mathrm{ha}^{-1}, 60 \mathrm{~kg} \mathrm{~K} \mathrm{O} \mathrm{ha}^{-1}, 40 \mathrm{~kg}$ FTE-BR-12 ha-1 (nível médio de adubação); 3) $70 \mathrm{~kg} \mathrm{~N} \mathrm{ha}^{-1}, 240$ $\mathrm{kg} \mathrm{P}_{2} \mathrm{O}_{5}$ ha $^{-1}, 120 \mathrm{~kg} \mathrm{~K}_{2} \mathrm{O} \mathrm{ha}^{-1}, 80 \mathrm{~kg}$ FTE-BR-12 ha-1 (nível alto de adubação); 4) nível médio de adubação + adubo verde (apenas o residual do cultivo de arroz) em que o tamanho de cada parcela foi de 10 x $5 \mathrm{~m}$. Antes da instalação do experimento, toda a área experimental recebeu calcário para elevar a saturação de base a 70\%, considerado valor ótimo para a cultura do feijoeiro; o calcário utilizado possuía as seguintes características: PRNT 74\%, $\mathrm{CaO} 49 \%$ e $\mathrm{MgO}$ 2,8\%. O detalhamento de metodologia pode ser encontrado no trabalho Fageria \& Baligar (1996).

Para se avaliar o efeito residual da adubação e da adubação aplicada no sulco de plantio, as parcelas $(10 \times 5 \mathrm{~m})$ foram divididas ao meio, originando oito parcelas em cada bloco, com dimensões de 5 x 4,5 m, com 0,5 $\mathrm{m}$ de espaço entre as parcelas divididas e os tratamentos consistiram em oito níveis de adubação: 1) testemunha, que foi dividida em duas, uma metade ficou como testemunha $\left(\mathrm{T}_{1}\right)$ e a outra recebeu $40 \mathrm{~kg} \mathrm{~N}^{-1}$ em cobertura $\left(\mathrm{T}_{2}\right) ; 2$ ) adubação média, em que uma metade ficou sem adubação para avaliar o efeito residual $\left(\mathrm{T}_{3}\right)$ e a outra recebeu $50 \mathrm{~kg} \mathrm{~N} \mathrm{ha}^{-1}$, $60 \mathrm{~kg} \mathrm{P}_{2} \mathrm{O}_{5}$ ha $^{-1}, 40 \mathrm{~kg} \mathrm{~K}_{2} \mathrm{O} \mathrm{ha}^{-1}\left(\mathrm{~T}_{4}\right) ; 3$ ) adubação alta, uma metade ficou para avaliar o efeito residual $\left(\mathrm{T}_{5}\right)$ e outra recebeu $25 \mathrm{~kg} \mathrm{~N}$ $\mathrm{ha}^{-1}, 30 \mathrm{~kg} \mathrm{P}_{2} \mathrm{O}_{5} \mathrm{ha}^{-1}, 20 \mathrm{~kg} \mathrm{~K}_{2} \mathrm{O} \mathrm{ha}^{-1}\left(\mathrm{~T}_{6}\right)$; 4) fertilidade média mais adubo verde, sendo que uma metade recebeu $75 \mathrm{~kg} \mathrm{~N}^{-1}$, $90 \mathrm{~kg} \mathrm{P}_{2} \mathrm{O}_{5}$ ha $^{-1}, 60 \mathrm{~kg} \mathrm{~K}_{2} \mathrm{O} \mathrm{ha}^{-1}\left(\mathrm{~T}_{7}\right)$ e a outra $100 \mathrm{~kg} \mathrm{~N} \mathrm{ha}^{-1}, 120 \mathrm{~kg}$ $\mathrm{P}_{2} \mathrm{O}_{5} \mathrm{ha}^{-1}, 80 \mathrm{~kg} \mathrm{~K}_{2} \mathrm{O} \mathrm{ha}^{-1}\left(\mathrm{~T}_{8}\right)$.

O delineamento experimental usado foi de blocos casualizados, com quatro repetições; o nitrogênio, o fósforo e o potássio foram aplicados na forma de sulfato de amônio, superfosfato triplo e cloreto de potássio, respectivamente.

Todas as adubações foram aplicadas no sulco de plantio, por ocasião da semeadura, em todos os anos de cultivo, com a exceção do $\mathrm{N}$, que foi parcelado. Nos tratamentos $\mathrm{T}_{2}, \mathrm{~T}_{4}$ e $\mathrm{T}_{6}$ metade do $\mathrm{N}$ foi aplicado no plantio, e o restante 42 dias após; nos tratamentos $\mathrm{T}_{7}$ e $\mathrm{T}_{8}$, um terço do $\mathrm{N}$ foi aplicado no plantio, um terço 42 dias após, e o restante na diferenciação do primórdio floral.

Utilizou-se a cultivar de arroz Metica 1, semeada em espaçamento de $20 \mathrm{~cm}$ entre linhas, numa densidade de 100 sementes por metro. Colheram-se as linhas centrais de cada parcela, deixando-se $0,5 \mathrm{~m}$ nas extremidades e $1 \mathrm{~m}$ nas laterais, como bordadura, resultando em uma área útil para cada parcela de 3 x 3,5 m (10,5 $\left.\mathrm{m}^{2}\right)$; na época da colheita, colheram-se plantas em $1 \mathrm{~m}$ de extensão na fileira em cada parcela, para determinar a produção de matéria seca e realizar a análise química. Para determinação dos teores de nutrientes, as plantas foram secadas em estufa a $70^{\circ} \mathrm{C}$, moídas e digeridas com mistura de ácido nítrico e perclórico 2:1; o $\mathrm{N}$ foi determinado pelo método de Kjeldahl, o $\mathrm{P}$ colorimetricamente, e os demais nutrientes por absorção atômica. Após a determinação da produção de grãos, foram feitas amostragens de grãos para análise química, utilizando-se a mesma metodologia de análise da parte aérea; a metodologia 
Tabela 3. Peso de 1000 grãos e esterilidade de espiguetas sob diferentes tratamentos de adubação ${ }^{1}$

\begin{tabular}{|c|c|c|c|c|c|c|c|c|}
\hline \multirow[b]{2}{*}{ Tratamentos ${ }^{2}$} & \multicolumn{2}{|c|}{$1^{\circ}$ ano } & \multicolumn{2}{|c|}{$2^{-0}$ ano } & \multicolumn{2}{|c|}{$3^{-}$ano } & \multicolumn{2}{|c|}{ Média } \\
\hline & $\begin{array}{c}\text { Peso de } \\
1000 \text { Grãos } \\
\text { (g) }\end{array}$ & $\begin{array}{l}\text { Esterilidade de } \\
\text { Espiguetas } \\
(\%)\end{array}$ & $\begin{array}{c}\text { Peso de } \\
1000 \text { Grãos } \\
(\mathrm{g})\end{array}$ & $\begin{array}{c}\text { Esterilidade de } \\
\text { Espiguetas } \\
(\%)\end{array}$ & $\begin{array}{c}\text { Peso de } \\
1000 \text { Grãos } \\
\text { (g) }\end{array}$ & $\begin{array}{l}\text { Esterilidade de } \\
\text { Espiguetas } \\
(\%)\end{array}$ & $\begin{array}{c}\text { Peso de } \\
1000 \text { Grãos } \\
\text { (g) }\end{array}$ & $\begin{array}{l}\text { Esterilidade de } \\
\text { Espiguetas } \\
(\%)\end{array}$ \\
\hline $\mathrm{T}_{2}$ & 25,80 & 30 & 26,35 & $29 \mathrm{ab}$ & $22,55 \mathrm{c}$ & 44 & $24,90 \mathrm{ab}$ & 34 \\
\hline $\mathrm{T}_{3}$ & 25,55 & 28 & 25,80 & $34 a$ & $23,93 \mathrm{abc}$ & 44 & $25,12 \mathrm{ab}$ & 35 \\
\hline $\mathrm{T}_{4}$ & 25,73 & 34 & 26,30 & $30 \mathrm{ab}$ & $23,45 \mathrm{abc}$ & 42 & $25,16 \mathrm{ab}$ & 35 \\
\hline $\mathrm{T}_{7}$ & 26,90 & 22 & 26,40 & $27 \mathrm{ab}$ & $24,73 \mathrm{a}$ & 45 & $26,01 \mathrm{a}$ & 31 \\
\hline $\mathrm{T}_{8}$ & 25,25 & 32 & 26,85 & $21 b$ & $23,60 \mathrm{abc}$ & 41 & $25,23 \mathrm{ab}$ & 31 \\
\hline Teste $\mathrm{F}(\mathrm{T})$ & ns & Ns & ns & $*$ & $* *$ & ns & $* *$ & ns \\
\hline Teste F(Ano) & & & & & & & $* *$ & $* *$ \\
\hline Teste $\mathrm{F}(\mathrm{T} \times \mathrm{A})$ & & & & & & & $*$ & $*$ \\
\hline
\end{tabular}

$*, * *, n s=$ significativo a 5 e $1 \%$ de probabilidade e não significativo, respectivamente

${ }^{1}$ Médias seguidas pela mesma letra, na mesma coluna, não diferem significativamente pelo teste de Tukey a $5 \%$ de probabilidade

${ }^{2} \mathrm{~T}_{1}=$ Testemunha, $\mathrm{T}_{2}=40 \mathrm{~kg} \mathrm{~N} \mathrm{ha}^{-1}$ em cobertura, $\mathrm{T}_{3}=$ Efeito residual da adubação média, $\mathrm{T}_{4}=$ Efeito residual da adubação média $+50 \mathrm{~kg} \mathrm{~N}^{-1}, 60 \mathrm{~kg} \mathrm{P}_{2} \mathrm{O}_{5}$ ha ${ }^{-1} \mathrm{e} 40 \mathrm{~kg} \mathrm{~K}_{2} \mathrm{O}^{-1} \mathrm{~T}^{-1}, \mathrm{~T}_{5}=$ Efeito residual da adubação alta, $\mathrm{T}_{6}=$ Efeito residual da adubação alta $+25 \mathrm{~kg} \mathrm{~N}$ ha $^{-1}, 30 \mathrm{~kg} \mathrm{P}_{2} \mathrm{O}_{5}$ ha $^{-1}$ e $20 \mathrm{~kg} \mathrm{~K}_{2} \mathrm{O}^{-1}$, $\mathrm{T}_{7}=$ Efeito residual da adubação média + efeito residual da adubação verde

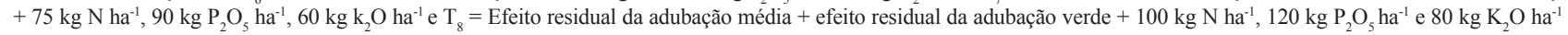

níveis de adubação e ano de cultivo para os parâmetros de peso de 1000 grãos e esterilidade de espiguetas, razão por que os dados referentes esses componentes são apresentados para cada ano agrícola (Tabela 3). Entre os componentes da produção, o número de panículas por $\mathrm{m}^{2}$ e o peso de 1000 grãos (média de três anos) foram significativamente influenciados pelos níveis de adubação, enquanto os maiores números de panícula por $\mathrm{m}^{2}$ foram obtidos nos tratamentos $\mathrm{T}_{4}$ (efeito residual a nível médio de adubação + adubação verde $+50 \mathrm{~kg} \mathrm{~N} \mathrm{ha}^{-1}, 60 \mathrm{~kg} \mathrm{P}_{2} \mathrm{O}_{5} \mathrm{ha}^{-1} \mathrm{e}$ $40 \mathrm{~kg} \mathrm{~K}_{2} \mathrm{O} \mathrm{ha}^{-1}$ ) e $\mathrm{T}_{7}$ (efeito residual a nível médio de adubação média $+75 \mathrm{~kg} \mathrm{~N} \mathrm{ha}^{-1}, 90 \mathrm{~kg}_{2} \mathrm{O}_{5} \mathrm{ha}^{-1}$, e $60 \mathrm{~kg} \mathrm{~K}_{2} \mathrm{O} \mathrm{ha}^{-1}$ ); as maiores produções de grãos também foram obtidas nesses tratamentos, ressaltando-se que o número de panículas em $\mathrm{T}_{4} \mathrm{e}$ $\mathrm{T}_{7}$ aumentou em 24 e 18\%, respectivamente, em comparação com a testemunha $\left(\mathrm{T}_{1}\right)$; outro componente da produção influenciado significativamente pelos níveis de adubação foi o peso de 1000 grãos, indicando que o aumento na produção de grãos, devido à adubação, está associado a esses dois componentes.

Os componentes de produção número de panículas $\mathrm{m}^{-2}$ $\left(\mathrm{r}=0,54^{* *}\right)$, peso de grãos por panícula $\left(\mathrm{r}=0,54^{* *}\right)$ e peso de 1000 grãos $\left(\mathrm{r}=0,35^{* *}\right)$ correlacionaram-se positiva e significativamente, com a produção de grãos,conforme Tabela 4. Tais resultados indicam possibilidade de se incrementar o número de panículas por unidade de área com a adubação e, conseqüentemente, de aumentar a produtividade do arroz irrigado em solo de várzea. Vários trabalhos mostram que o número de panículas correlaciona-se positivamente com a produção de grãos na cultura de arroz (Yoshida, 1981; Gravois
\& Helms, 1992; Hasegawa et al., 1994); a cobertura com $40 \mathrm{~kg} \mathrm{~N}$ ha $^{-1}$ aumentou o número de panículas em 10\% em comparação com a testemunha, enquanto o aumento média da produção de grãos foi de $21 \%$ e o número de panículas por unidade de área possui relação positiva com a nutrição nitrogenada na cultura de arroz irrigado (Yoshida, 1981). A esterilidade de grãos também influencia a produtividade de grãos, ressaltando-se que nos primeiro e segundo anos, a esterilidade de espiguetas foi mínima sob o tratamento $T_{7}$, mas apresentou correlação negativa e significativa em relação ao comprimento de panícula $\left(\mathrm{r}=-0,45^{* *}\right)$, peso de 1000 grãos $(\mathrm{r}=-0,971 * *)$ e peso de grãos por panícula $(\mathrm{r}=-0,42 * *)$.

$\mathrm{Na}$ Tabela 5 são apresentados os valores médios de acumulação de nutrientes na parte aérea e nos grãos. Na parte aérea, a acumulação de nutrientes decresceu na seguinte ordem: $\mathrm{K}>\mathrm{N}>\mathrm{Ca}>\mathrm{P}>\mathrm{Mg}$, para os macronutrientes e $\mathrm{Mn}>\mathrm{Fe}>\mathrm{Zn}>$ $\mathrm{Cu}$, para os micronutrientes, porém nos grãos a acumulação decresceu na seguinte ordem: $\mathrm{N}>\mathrm{K}>\mathrm{P}>\mathrm{Mg}>\mathrm{Ca}$, entre os macronutrientes, e $\mathrm{Fe}>\mathrm{Mn}>\mathrm{Zn}>\mathrm{Cu}$, entre os micronutrientes. Fageria \& Baligar (1996) obtiveram seqüências semelhantes de acumulação de macro e de micronutrientes na parte aérea e nos grãos na cultura de arroz irrigado. Para produzir $5.500 \mathrm{~kg} \mathrm{ha}^{-1} \mathrm{de}$ grãos, a cultura de arroz irrigado acumula em média, na parte aérea e nos grãos, $100 \mathrm{~kg} \mathrm{~N} \mathrm{ha}^{-1}, 23 \mathrm{~kg} \mathrm{P} \mathrm{ha}^{-1}, 144 \mathrm{~kg} \mathrm{~K} \mathrm{ha}^{-1}, 18 \mathrm{~kg}$ $\mathrm{Ca} \mathrm{ha}^{-1}, 14 \mathrm{~kg} \mathrm{Mg} \mathrm{ha}{ }^{-1}, 339 \mathrm{~g} \mathrm{Zn} \mathrm{ha}^{-1}, 104 \mathrm{~g} \mathrm{Cu} \mathrm{ha}^{-1}, 1.757 \mathrm{~g} \mathrm{Fe} \mathrm{ha}^{-1}$ e $2.570 \mathrm{~g} \mathrm{Mn} \mathrm{ha}^{-1}$.

Em relação à distribuição de nutrientes na parte aérea e nos grãos de arroz, em média $55 \%$ do N, $63 \%$ do $\mathrm{P}, 13 \%$ do K, $11 \%$ do Ca, $50 \%$ do $\mathrm{Mg}, 34 \%$ do $\mathrm{Zn}, 90 \%$ do $\mathrm{Cu}, 42 \%$ do Fe e $10 \%$ do $\mathrm{Mn}$

Tabela 4. Coeficiente de correlação entre produção de matéria seca, produção de grãos e componentes de produção

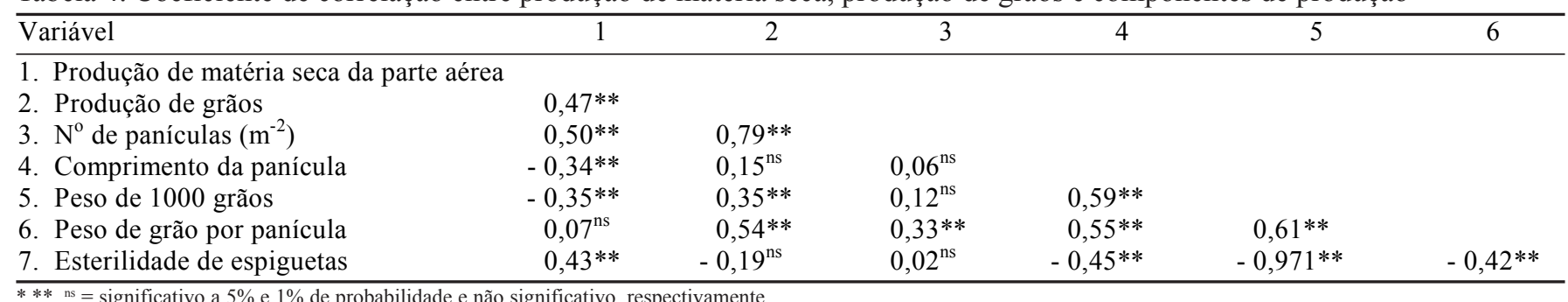


Tabela 5. Acumulação de nutrientes na parte aérea e nos grãos de arroz irrigado sob diferentes tratamentos de adubação ${ }^{1}$

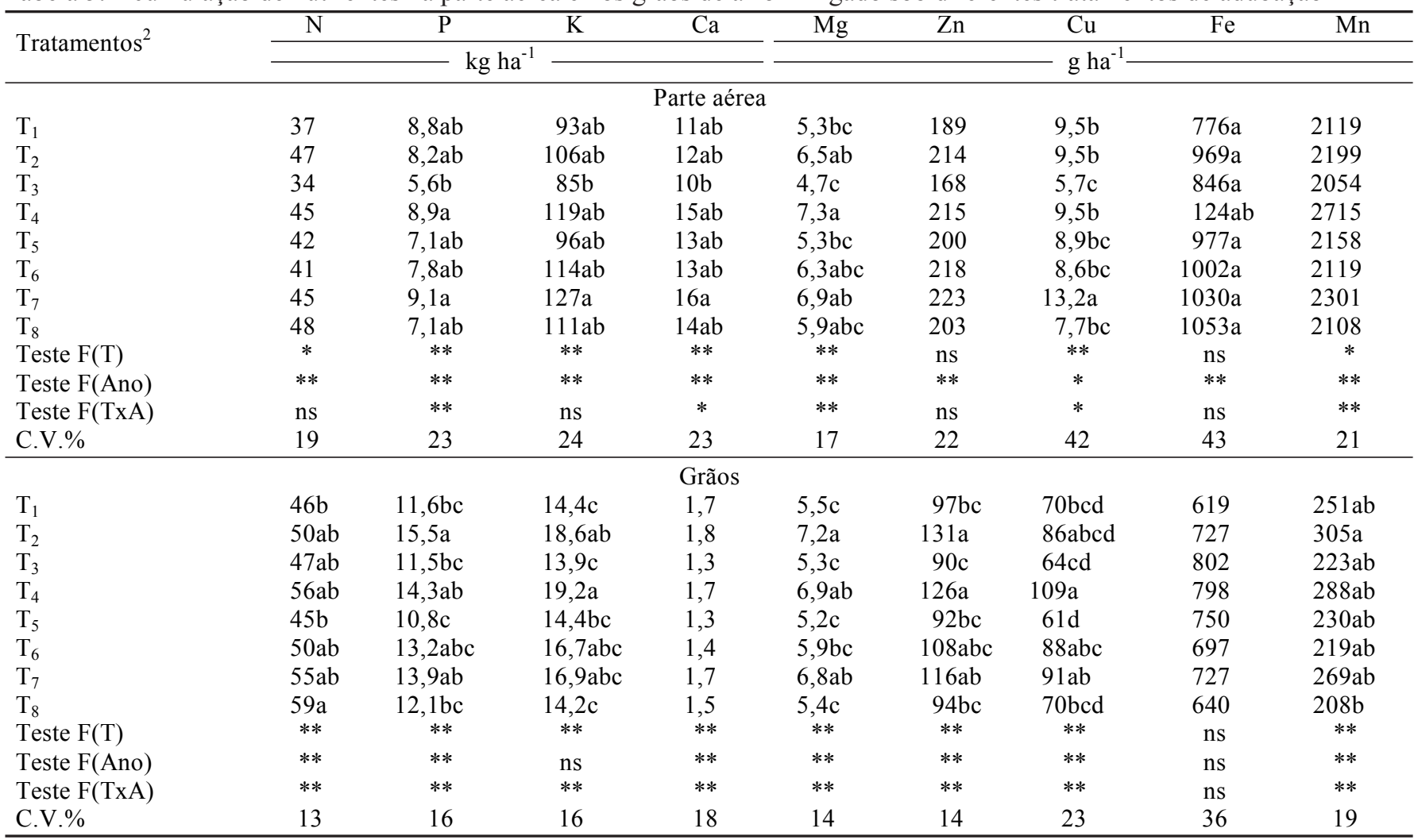

$*, * *$, ns $=$ significativo a $5 \%$ e $1 \%$ de probabilidade e não significativo, respectivamente

${ }^{1}$ Médias seguidas pela mesma letra na mesma coluna, não diferem significativamente pelo teste de Tukey, a $5 \%$ de probabilidade. Os valores são médias de três anos

${ }^{2} \mathrm{~T}_{1}=$ Testemunha, $\mathrm{T}_{2}=40 \mathrm{~kg} \mathrm{~N}$ ha $^{-1}$ em cobertura, $\mathrm{T}_{3}=$ Efeito residual da adubação média, $\mathrm{T}_{4}=$ Efeito residual da adubação média $+50 \mathrm{~kg} \mathrm{~N} \mathrm{ha-1}, 60 \mathrm{~kg} \mathrm{P}_{2} \mathrm{O}_{5}$ ha ${ }^{-1} \mathrm{e} 40 \mathrm{~kg} \mathrm{~K} \mathrm{O}_{2} \mathrm{ha}^{-1}, \mathrm{~T}_{5}=\mathrm{Efeito}$ residual da adubação alta, $\mathrm{T}_{6}=$ Efeito residual da adubação alta $+25 \mathrm{~kg} \mathrm{~N}^{-1}, 30 \mathrm{~kg} \mathrm{P}_{2} \mathrm{O}_{5}$ ha $^{-1} \mathrm{e} 20 \mathrm{~kg} \mathrm{~K}_{2} \mathrm{O}^{-1}$, $\mathrm{T}_{7}=$ Efeito residual da adubação média + efeito residual da adubação verde $+75 \mathrm{~kg} \mathrm{~N} \mathrm{ha}^{-1}, 90 \mathrm{~kg} \mathrm{P}_{2} \mathrm{O}_{5}$ ha $^{-1}, 60 \mathrm{~kg} \mathrm{k}_{2} \mathrm{O} \mathrm{ha}^{-1} \mathrm{e} \mathrm{T}_{8}={\text { Efeito residual da adubação média }+ \text { efeito residual da adubação verde }+100 \mathrm{~kg} \mathrm{~N}^{-1}, 120 \mathrm{~kg} \mathrm{P}_{2} \mathrm{O}_{5} \text { ha }{ }^{-1} \text { e } 80 \mathrm{~kg} \mathrm{~K}_{2} \mathrm{O} \text { ha }}^{-1}$

foram acumulados nos grãos, e o restante na parte aérea. Fageria et al. (1997) relataram as distribuições de macro e micronutrientes nas mesmas proporções na parte aérea e nos grãos, em cultivo de arroz irrigado em solo de várzea.

\section{CONCLUSÕES}

1. A aplicação de adubo no sulco de plantio aumenta significativamente a produção de matéria seca e de grãos do arroz irrigado.

2. A produção máxima de grãos é obtida com o tratamento de efeito residual de adubação média mais adubo verde $+75 \mathrm{~kg}$ $\mathrm{Nha}^{-1}, 90 \mathrm{~kg} \mathrm{P}_{2} \mathrm{O}_{5}$ ha $^{-1}$ e $60 \mathrm{~kg} \mathrm{~K}_{2} \mathrm{O} \mathrm{ha}^{-1}$.

3.As produtividades dos tratamentos que não recebem adubação no sulco de plantio não diferem da testemunha.

4. Entre os componentes da produção, o número de panículas por $\mathrm{m}^{2}$ foi o que apresentou maior coeficiente de correlação com as produções de matéria seca da parte aérea e de grãos.

\section{REFERÊNCIAS BIBLIOGRÁFICAS}

EMBRAPA. Serviço Nacional de Levantamento e Conservação de Solos (Rio de Janeiro). Manual de métodos de análise de solo. 2.ed. Rio de Janeiro, 1997. 212p.

FAGERIA, N.K. Maximizing crop yields. New York: Marcel Dekker, 1992.274p.

FAGERIA, N.K. Nutrição mineral. In: Vieira, N.R.A.; Santos A.B. dos; Santana, E.P. (eds.) A cultura do arroz no Brasil. Embrapa Arroz e Feijão, Santo Antonio de Goiás, GO, 1999a. p.172-196.
FAGERIA, N. K. Adubação e calagem. . In: Vieira, N.R.A.; Santos, A.B. dos; Santana, E.P. (eds.) A cultura do arroz no Brasil. Embrapa Arroz e Feijão, Santo Antonio de Goiás, GO, 1999b. p.329-353.

FAGERIA, N.K.; BALIGAR, V.C. Response of lowland rice and common bean grown in rotation to soil fertility levels on a varzea soil. Fertilizer Research, Dordrecht, v.45, p.13-20, 1996.

FAGERIA, N.K.; BALIGAR, V.C.; WRIGHT, R.J; CARVALHO, J.R. Lowland rice response to potassium fertilization and its effect on N and P uptake. Fertilizer Research, Dordrecht, v.21, p.157-162, 1990.

FAGERIA, N.K.; BARBOSA FILHO, M.P.; ZIMMERMANN, F.J.P. Caracterização química e granulométrica de solos de várzea de alguns Estados brasileiros. Pesquisa Agropecuária Brasileira, Brasília, v.29, n.2, p.267-274, 1994.

FAGERIA, N.K.; FERREIRA, E.; PRABHU, A.S.; BARBOSA FILHO, M.P.; FILIPPI, M.C. Seja o doutor do seu arroz. Piracicaba: Potafós, 1995. 20p. Potafós. Arquivo do Agrônomo, 9.

FAGERIA, N.K.; SANTOS, A.B. dos. Adubação fosfatada para o feijoeiro em solo de várzea. Revista Brasileira de Engenharia Agrícola e Ambiental, Campina Grande, v.2, n.2, p.124-127, 1998.

FAGERIA, N.K.; SANTOS, A B. dos; BALIGAR, V.C. Phosphorus soil test calibration for lowland rice on an Inceptisol. Agronomy Journal, Madison, v.89, n.5, p.737-742, 1997. 
FAGERIA, N.K.; STONE, L.F.; SANTOS, A.B. dos. Maximização da eficiência de produção das culturas. EMBRAPA, Brasília, DF, 1999.294p.

FAGERIA, N.K.; WRIGHT, R.J.; BALIGAR, V.C.; SOUSA, C.M. de. Characterization of physical and chemical properties of varzea soils of Goias State of Brazil. Communication in Soil Science and Plant analysis, New York, v.22, n.15, p.1631-1646, 1991.

GRAVOIS, K.A.; HELMS, R.S. Path analysis of rice yield and yield components as affected by seeding rate. Agronomy Journal, Madison, v.84, n.1, p.1-4, 1992.
HASEGAWA, T.; KORODA, Y.; SELIGMAN, G.; HORIE, T. Response of spikelet number to plant nitrogen concentration and dry weight in paddy rice. Agronomy Journal, Madison, v.86, n.4, p.673-676, 1994.

MORAES, J.F.V; RABELO, N.A. Um método simples para a digestão de amostras de plantas. Goiânia: EMBRAPACNPAF, 1986. 12p. EMBRAPA-CNPAF. Documentos, 12

van RAIJ, B. Fertilidade do solo e adubação. Piracicaba: Ceres, 1991.343p.

YOSHIDA, S. Fundamentals of rice crop science. Los Baños: IRRI, 1981.269p. 